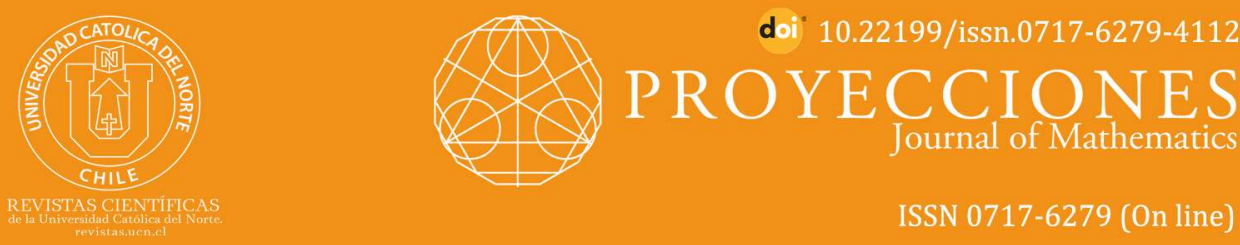

\title{
Line graph of unit graphs associated with finite commutative rings
}

Pranjali ${ }^{1}$ (1) orcid.org/0000-0002-1106-5083

Amit Kumar ${ }^{2}$ (i) orcid.org/0000-0002-3919-3423

Pooja Sharma ${ }^{3}$ @ orcid.org/0000-0001-9110-8348

${ }^{1}$ University of Rajasthan, Dept. of Mathematics. Jaipur, RJ, India.

v pranjali48@gmail.com

Banasthali Vidyapith, Dept. of Mathematics and Statistics. Banasthali, RJ, India.

2匹amitsu48@gmail.com; ${ }^{3 凶}$ sapooja1984@gmail.com

Received: 2020/04/21 | Accepted: 2021/01/09

Abstract:

For a given graph $G$, its line graph denoted by $L(G)$ is a graph whose vertex set $V(L(G))=E(G)$ and $\left\{e_{1}, e_{2}\right\} \in E(L(G))$ if $e_{1}$ and $e_{2}$ are incident to a common vertex in $G$. Let $R$ be a finite commutative ring with nonzero identity and $G(R)$ denotes the unit graph associated with $R$. In this manuscript, we have studied the line graph $L(G(R)$ ) of unit graph $G(R)$ associated with $R$. In the course of the investigation, several basic properties, viz., diameter, girth, clique, and chromatic number of $L(G(R))$ have been determined. Further, we have derived sufficient conditions for $L(G(R))$ to be Planar and Hamiltonian.

Keywords: Commutative rings; Unit graph; Clique; Chromatic number; Planarity; Hamiltonian.

MSC (2020): 13A99; 05C25, 05C69; 05C15.

\section{Cite this article as (IEEE citation style):}

Pranjali, A. Kumar, and P. Sharma, "Line graph of unit graphs associated with finite commutative rings", Proyecciones (Antofagasta, On line), vol. 40, no. 4, pp. 919-926, 2021, doi: 10.22199/issn.0717-6279-4112

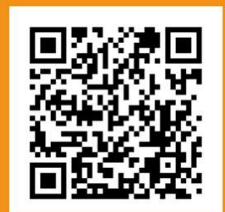

Article copyright: (C) 2021 Pranjali, Amit Kumar and Pooja Sharma. This is an open access article distributed under the terms of the Creative Commons License, which permits unrestricted use and distribution provided the original author and source are credited.

(cc) BY 


\section{Introduction}

Let $n$ be a positive integer and $\mathbf{Z}_{n}$ be the ring of integers modulo $n$. In 1990, Grimaldi [3] introduced the notion of unit graph denoted as $G\left(\mathbf{Z}_{n}\right)$ based on the elements of $\mathbf{Z}_{n}$ and two distinct vertices $x$ and $y$ are adjacent if and only if $x+y$ is a unit of $\mathbf{Z}_{n}$. This investigation was further continued by Ashrafi et al. [2], where author's were interested to generalize the unit graph $G\left(\mathbf{Z}_{n}\right)$ to $G(R)$ for an arbitrary associative ring $R$. Now a days, there is an enormous amount of literature built up on several parameters of unit graphs. It would be too unwieldily to cite all references, but a few pioneering papers like $[1,8]$ are worth comprehension.

The following result provides some insight into the structure of unit graphs, which have been established in [2] and is found useful to derive new results.

Lemma 1.1. Let $R$ be a finite commutative ring. Then for $G(R)$ the following statements hold:

(i) If $2 \notin U(R)$, then $G(R)$ is $|U(R)|$-regular;

(ii) If $2 \in U(R)$, then $G(R)$ is $(|U(R)|,|U(R)|-1)$-semi regular.

The benefit of studying of $L(G)$ associated with $G$ is that one may recover the structure of any connected graph from its line graph, i.e., there is a one-to-one correspondence between the class of connected graphs and the class of associated line graphs. With the class of line graph of unit graphs keeping in hand, it is natural to keep an eye on the properties of unit graphs and determine the properties of their line graphs. In this paper, we determine several basic properties of line graph $L(G(R))$ of unit graphs $G(R)$ associated with finite commutative rings $R$. In particular, our focus will be placed on diameter, girth, clique, and chromatic number of $L(G(R))$.

Throughout, the paper we shall consider only simple graphs, viz., graphs in which any two vertices are joined by at most one edge, and without self-loops and $R$ will be a finite commutative ring with $1 \neq 0$. As usual, $\mathbf{Z}, \mathbf{Z}_{n}$, and $\mathbf{F}_{q}$ will denote the ring of integers, the ring of integers modulo $n$, and a finite field with $q$ elements, respectively. We will usually use the notation " $\mathbf{Z}_{2}^{t}$ " for " $\underbrace{\mathbf{Z}_{2} \times \mathbf{Z}_{2} \times \mathbf{Z}_{2} \times \cdots \times \mathbf{Z}_{2}}_{(\text {-times })}$ "

and " $\cup_{i=1}^{t} G$ " for $t$-copies of graph $G$. We further denote the characteristic of ring $R$ by $C h(R)=p$. To avoid trivialities, we implicitly assume when necessary that graphs are nonempty. For all terminology and notations in graph theory and abstract algebra, not specifically mentioned or defined in this paper, we refer the reader to the standard textbooks [4] and [5], respectively.

The following lemma established in [7] will be used frequently to derive new results.

Lemma 1.2. Let $R \cong \mathbf{Z}_{2}^{t} \times S, t \geq 0$. Then $G(R)$ has $2^{t}$-copies of $G(S)$, where $S$ is isomorphic to any one of the rings $\mathbf{Z}_{2}, \mathbf{Z}_{4}, \mathbf{Z}_{2}[X] /\left\langle X^{2}\right\rangle$, and $\mathbf{Z}_{6}$. 


\section{Basic Notations and Results}

This section is devoted to study the basic properties of line graph of unit graphs associated with finite commutative ring. Our ultimate goal is to establish the sufficient conditions for $L(G(R))$ to be Planar and Hamiltonian.

Definition 2.1. The line graph $L(G)$ of a graph $G$, is a graph defined by $V(L(G)):=$ $E(G)$ and $\left\{e_{1}, e_{2}\right\} \in E(L(G))$ if $e_{1}$ and $e_{2}$ are incident to a common vertex in $G$. If $\{x, y\} \in E(G)$ we will denote the corresponding vertex of $L(G)$ by $[x, y]$.

Let $R$ be a commutative ring with nonzero identity and $U(R)$ be the set of unit elements of $R$. For the convenience, note that the line graph $L(G(R))$ of unit graph $G(R)$ of $R$ will have vertices of the form $[u, v]$ in which the sum of $u$ and $v$ is a unit of $R$, i.e., $u+v \in U(R)$.

An example of unit graph and its corresponding line graph over finite field is shown in Fig. 1.

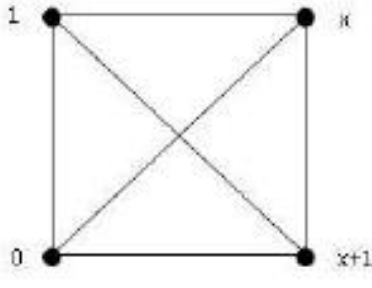

$\mathrm{G}\left(\mathrm{F}_{4}\right)$

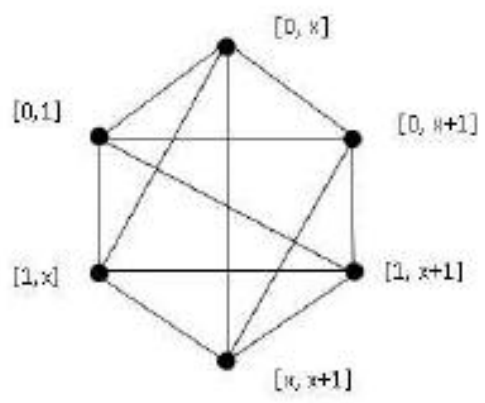

$L\left(G\left(F_{4}\right)\right.$

Fig. 1. The unit graph $G\left(\mathbb{F}_{4}\right)$ and the line graph $L\left(G\left(\mathbb{F}_{4}\right)\right)$

It is easy to see that for given rings $R_{1}$ and $R_{2}$, if $R_{1} \cong R_{2}$, then $G\left(R_{1}\right) \cong$ $G\left(R_{2}\right)$, and hence $L\left(G\left(R_{1}\right)\right) \cong L\left(G\left(R_{2}\right)\right)$ as for instance; for $\mathbf{Z}_{2} \times \mathbf{Z}_{3} \cong \mathbf{Z}_{6}$, clearly $G\left(\mathbf{Z}_{2} \times \mathbf{Z}_{3}\right) \cong G\left(\mathbf{Z}_{6}\right) \cong C_{6}$ and $L\left(G\left(\mathbf{Z}_{2} \times \mathbf{Z}_{3}\right)\right) \cong L\left(G\left(\mathbf{Z}_{6}\right)\right) \cong C_{6}$. But it might be possible that if $R_{1} \neq R_{2}$, still $G\left(R_{1}\right) \cong G\left(R_{2}\right)$, and then $L\left(G\left(R_{1}\right)\right) \cong L\left(G\left(R_{2}\right)\right)$, as for instance; $G\left(\frac{\mathbf{Z}_{2}[x]}{\left\langle x^{2}\right\rangle}\right) \cong G\left(\mathbf{Z}_{4}\right) \cong C_{4}$ and $L\left(G\left(\frac{\mathbf{Z}_{2}[x]}{\left\langle x^{2}\right\rangle}\right)\right) \cong L\left(G\left(\mathbf{Z}_{4}\right)\right) \cong C_{4}$.

Theorem 2.1. The following are well-known results:

i) If a graph $G$ is connected, then $L(G)$ is connected and vice-versa.

ii) If a graph $G$ contains a star graph with ' $n$ ' vertices, then its line graph will contain a clique of size ' $n-1$ '.

Lemma 2.2. [4, Page 71] Let $G$ be an arbitrary graph and $L(G)$ be its line graph. Then for any vertex $[u, v] \in V[L(G)]$ the degree is given by

$$
\operatorname{deg}_{L(G)}[u, v]=\operatorname{deg}_{G}(u)+\operatorname{deg}_{G}(v)-2
$$


As in the case of unit graphs, there are two specific kinds in structure either regular or semi-regular, but which fails to be in case of corresponding line graph, so it is necessary to measure this deviation. This has been carried out in the next important result.

Theorem 2.3. Let $R$ be a finite commutative ring with unity and let $G(R)$ and $L(G(R))$ be unit graph, and its line graph, respectively associated with $R$. Then for any vertex $\left[v_{1}, v_{2}\right] \in V[L(G(R))]$ the following statements hold:

i) If $2 \notin U(R)$, then $L(G(R))$ is $(2 \cdot|U(R)|-2)$-regular;

ii) If $2 \in U(R)$, then $\operatorname{deg}\left[v_{1}, v_{2}\right] \in\{(2|U(R)|-3),(2|U(R)|-4),(2|U(R)|-2)\}$.

Proof. Let $G(R)$ be unit graph and $L(G(R))$ be its line graph. Then there are two possibilities.

If $2 \notin U(R)$, then in view of Lemma $1.1, G(R)$ is $|U(R)|$-regular. Take $\left[v_{1}, v_{2}\right]$ be an arbitrary vertex of $L(G(R))$. Then by Lemma 2.2

$\operatorname{deg}\left[\mathrm{v}_{1}, v_{2}\right]=2 \cdot|U(R)|-2$.

Next if $2 \in U(R)$, then in view of Lemma 1.1, the degree of each vertex $v$ is either $|U(R)|$ or $|U(R)|-1$ in $G(R)$. Let $\left[v_{1}, v_{2}\right]$ be an arbitrary vertex of $L(G(R))$ to determine the degree of $\left[v_{1}, v_{2}\right]$, we shall make use of the Lemma 1.1 and Lemma 2.2 in each of the following cases :

Case $i)$ If $v_{1}$ and $v_{2} \in U(R)$, then $\operatorname{deg}\left[\mathrm{v}_{1}, v_{2}\right]=2 \cdot|U(R)|-4$.

Case $i i$ ) If $v_{1} \in Z(R)$ and $v_{2} \in U(R)$ or (vice-versa), then $\operatorname{deg}\left[\mathrm{v}_{1}, v_{2}\right]=2 \cdot|U(R)|-3$.

Case $i i i)$ If $v_{1}$ and $v_{2} \in Z(R)$, then

$\operatorname{deg}\left[\mathrm{v}_{1}, v_{2}\right]=2 \cdot|U(R)|-2$.

Therefore from the above cases we get the desired result.

Theorem 2.4. Let $R$ be a finite commutative ring with unity and $U(R)$ be the set of units of $R$. Then the following statements hold:

i) If $2 \notin U(R)$, then $|V(L(G(R)))|=\frac{1}{2}|R| \cdot|U(R)|$;

ii) If $2 \in U(R)$, then $|V(L(G(R)))|=\frac{1}{2}(|R|-1) \cdot|U(R)|$.

Proof. Let $R$ be a finite commutative ring with unity and $U(R)$ be the set of units of $R$. First, if $2 \notin U(R)$, then $G(R)$ is $|U(R)|$-regular, and hence the number of edges in $G(R)$ is equal to $\frac{1}{2}|R| \cdot|U(R)|$. Thus $|V(L(G(R)))|=\frac{1}{2}|R| \cdot|U(R)|$.

Next if $2 \in U(R)$, then by Lemma $1.1, G(R)$ is $(|U(R)|,|U(R)|-1)$ semiregular, i.e., for every $v \in U(R), \operatorname{deg}(v)=|U(R)|-1$ and for every $v \in R \backslash U(R)$, $\operatorname{deg}(v)=|U(R)|$. Therefore, by Hand-shaking lemma, the number of edges in $G(R)$ is equal to $\frac{1}{2}(|R|-1) \cdot|U(R)|$, and hence $|V(L(G(R)))|=\frac{1}{2}(|R|-1) \cdot|U(R)|$. 
Definition 2.2. For distinct vertices $x$ and $y$ of $G$, let $d(x, y)$ be length of shortest path from $x$ to $y$. If there is no any path between $x$ and $y$, then we write $d(x, y)=\infty$. Then the diameter of graph denoted by $\operatorname{diam}(G)$ and is defined as $\operatorname{diam}(\mathrm{G})=\sup \{d(x, y): x, y \in V(G)\}$

One can easily notice that if $R \cong \mathbf{Z}_{4}$, then $\operatorname{diam}(G(R))$ and $\operatorname{diam}(L(G(R)))$ are both equal to 2 . However, if $R \cong \mathbf{Z}_{2} \times \mathbf{Z}_{2}$, then $\operatorname{diam}(G(R))$ and $\operatorname{diam}(L(G(R)))$ is equal to infinite.

Theorem 2.5. Let $R$ be a finite commutative ring, $G(R)$ be unit graph and $L(G(R))$ be its line graph. Then

i) If $R$ is a field, then $\operatorname{diam}(L(G(R))) \in\{1,2\}$;

ii) If $R$ is local but not field and have $\mathbf{Z}_{2}$ as a quotient, then $\operatorname{diam}(L(G(R)))=2$;

iii) If $R$ is local but not field and does not have $\mathbf{Z}_{2}$ as a quotient, then $\operatorname{diam}(L(G(R)))=$ 3.

Proof. $i$ ) Let $R$ be a finite field of characteristic $p$. If $p=2$ and $a, b$ be two nonzero distinct elements of $\mathbf{F}_{2^{k}}$, then $[0, a]-[0, b]-[1, b]$ is a path of length 2 in $L(G(R))$. On the other hand, if $p>2$ (but $R \mathbf{Z}_{3}$ ), then for two arbitrary vertices $\left[v_{m}, v_{n}\right]$ and $\left[v_{k}, v_{l}\right]$ of $L\left(G\left(\mathbf{F}_{p^{k}}\right)\right)$ one have a path $\left[v_{m}, v_{n}\right]-\left[v_{m}, v_{k}\right]-\left[v_{k}, v_{l}\right]$ (or $\left.\left[v_{m}, v_{n}\right]-\left[v_{n}, v_{l}\right]-\left[v_{k}, v_{l}\right]\right)$. Accordingly $v_{m}+v_{k} \neq p^{k}$ or $v_{n}+v_{l} \neq p^{k}$, which again gives a path of length 2 . Therefore $\operatorname{diam}(L(G(R)))=2$.

Now if $R \cong \mathbf{Z}_{3}$, then $L(G(R)) \cong K_{2}$, for which $\operatorname{diam}(L(G(R)))=1$.

ii) Next, let $R$ be local with $\langle m\rangle$ as a maximal ideal, which is not field and have $\mathbf{Z}_{2}$ as a quotient, then in view of [2, Theorem 3.2], $G(R)$ is complete bipartite with $\langle m\rangle$ and $R \backslash\langle m\rangle$ as two partite sets. In $L(G(R))$ for any two arbitrary vertices $\left[m_{1}, u_{1}\right],\left[m_{2}, u_{2}\right]$ one have the following path $\left[m_{1}, u_{1}\right]-\left[m_{1}, u_{2}\right]-\left[m_{2}, u_{2}\right]$ (or $\left[m_{1}, u_{1}\right]-\left[m_{2}, u_{1}\right]-\left[m_{2}, u_{2}\right]$ ) of length 2. Therefore, $\operatorname{diam}(L(G(R)))=2$.

iii) Let $R$ be local with maximal ideal $\langle m\rangle$, which is not field and does not have $\mathbf{Z}_{2}$ as a quotient. Then clearly no two elements of $\langle m\rangle$ are adjacent. However the elements of $\langle m\rangle$ are adjacent with elements of $R \backslash\langle m\rangle$ in $G(R)$. Let $u_{1}$, $u_{2}$ be two elements of $R \backslash\langle m\rangle$ which are adjacent in $G(R)$, then for distinct vertices $\left[u_{1}, u_{2}\right],\left[u_{3}, u_{4}\right] \in V(L(G(R)))$ one can see the presence of path of length 3 namely, $\left[u_{1}, u_{2}\right]-\left[m_{1}, u_{1}\right]-\left[m_{2}, u_{3}\right]-\left[u_{3}, u_{4}\right]$, where $m_{1}, m_{2} \in\langle m\rangle$. Therefore, $\operatorname{diam}(L(G(R)))=3$.

Definition 2.3. For a graph $G$, the girth of $G$ denoted by $\operatorname{gir}(G)$ is the length of smallest cycle in $G$. If $G$ does not contain any cycle, then $\operatorname{gir}(G)=\infty$.

Theorem 2.6. Let $R$ be a finite commutative ring with unity. If $|U(R)| \geq 3$, then $\operatorname{gir}(L(G(R)))=3$.

Proof. Let $R$ be a finite commutative ring with unity such that $|U(R)| \geq 3$. If $R$ has atleast 3 units (say $u_{1}, u_{2}, u_{3}$ ), then corresponding to these units $u_{1}, u_{2}$ and $u_{3}$ there 
exist three vertices namely, $\left[0, u_{1}\right],\left[0, u_{2}\right]$ and $\left[0, u_{3}\right]$ in $L(G(R))$, which are mutually adjacent. Therefore in $L(G(R))$ one can easily find a triangle, which ensures that $\operatorname{gir}(L(G(R)))=3$.

Theorem 2.7. Let $R$ be a finite local ring with nonzero maximal ideal $\langle m\rangle$ and $R \neq \mathbf{Z}_{4}$. Then $\operatorname{gir}(L(G(R)))=3$.

Proof. Let $R$ be a finite local ring with nonzero maximal ideal $\langle m\rangle$. Then $\langle m\rangle=$ $\operatorname{ann}(x)$ for some $x \in\langle m\rangle^{*}$. If there are distinct $y, z \in\langle m\rangle^{*} \backslash\{x\}$ with $y+z \in U(R)$, then $y-x-z-y$ is a cycle of length three in $G(R)$, and hence there is a associated 3 cycle in $L(G(R))$. This implies that $\operatorname{gir}(L(G(R)))=3$. On the other hand if $G(R)$ do not contain such triangle, then we may choose $u_{i} \in U(R)$, which corresponds a triangle namely, $\left[0, u_{1}\right]-\left[0, u_{2}\right]-\left[0, u_{3}\right]-\left[0, u_{1}\right]$ in $L(G(R))$. Hence $\operatorname{gir}(L(G(R)))=3$.

The following result is especially interesting and have appeared in [1].

Proposition 2.8. Let $R$ be a finite commutative ring with unity having $|U(R)| \leq 2$. Then $R$ is isomorphic to $\mathbf{Z}_{2}^{t} \times S(t \geq 0)$, where $S$ is isomorphic to $\mathbf{Z}_{2}$ or $\mathbf{Z}_{3}$ or $\mathbf{Z}_{4}$ or $\mathbf{Z}_{6}$ or $\frac{\mathbf{Z}_{2}[x]}{\left\langle x^{2}\right\rangle}$.

Theorem 2.9. Let $R$ be a finite commutative ring with unity having $|U(R)| \leq 2$. Then $\operatorname{gir}(L(G(R))) \in\{4,6, \infty\}$.

Proof. Let $R$ be a finite commutative ring with unity such that $|U(R)| \leq 2$. Then in view of Proposition 2.8, $R$ is isomorphic to $\mathbf{Z}_{2}^{t} \times S(t \geq 0)$, where $S \cong \mathbf{Z}_{2}$ or $\mathbf{Z}_{3}$ or $\mathbf{Z}_{4}$ or $\mathbf{Z}_{6}$ or $\frac{\mathbf{Z}_{2}[x]}{\left\langle x^{2}\right\rangle}$. First, when $S \cong \mathbf{Z}_{2}$, then the unit graph $G(R) \cong \cup_{i=1}^{2^{t}} K_{2}$, and its corresponding line graph $L(G(R)) \cong \cup_{i=1}^{2^{t}} K_{1}$. Therefore $\operatorname{gir}(L(G(R)))=\infty$.

Next, if $S \cong \mathbf{Z}_{3}$, then for $t=0, \operatorname{gir}(L(G(R)))=\infty$, however for $t>0$ the girth of $L(G(R))$ can be determined analogues to those used for $S \cong \mathbf{Z}_{6}$.

Now, when $S \cong \mathbf{Z}_{4}$ or $\mathbf{Z}_{6}$ or $\frac{\mathbf{Z}_{2}[x]}{\left\langle x^{2}\right\rangle}$. Then in view of [2, Theorem 3.2.], $G(R)$ is cycle if and only if $R \cong \mathbf{Z}_{4}$ or $\mathbf{Z}_{6}$ or $\frac{\mathbf{Z}_{2}[x]}{\left\langle x^{2}\right\rangle}$, and hence in all the cases $L(G(R)) \cong G(R)$. In light of Lemma 1.2, $G\left(\mathbf{Z}_{2}^{t} \times S\right)$ is isomorphic to $2^{t}$-copies of $G(S)$, and hence $L\left(G\left(\mathbf{Z}_{2}^{t} \times S\right)\right)$ is isomorphic to $2^{t}$-copies of $L(G(S))$. Therefore for each of the above listed rings girth of $L(G(R))$ is either 4 or 6 . Thus $\operatorname{gir}(L(G(R))) \in\{4,6, \infty\}$.

Theorem 2.10. Let $R$ be finite commutative ring with unity and let $L(G(R))$ be its line graph of unit graph $G(R)$. Then $\operatorname{gir}(L(G(R))) \in\{3,4,6, \infty\}$.

Proof. Invoking Theorem 2.6 and Theorem 2.9, the proof follows.

Theorem 2.11. Let $R$ be finite commutative ring with unity such that $|R| \geq 4$. If $R$ does not have $\mathbf{Z}_{2} \times \mathbf{Z}_{2}$ as a quotient, then $L(G(R))$ is Hamiltonian. 
Proof. Let $R$ be a finite commutative ring with unity such that $|R| \geq 4$ and let $G(R)$ be unit graph associated with $R$. Assume that $R$ does not have $\mathbf{Z}_{2} \times \mathbf{Z}_{2}$ as a quotient, then in this case $G(R)$ is connected and in view of [2] it is known that $G(R)$ is Hamiltonian. But in light of [4, Theorem 8.8] it is clear that "if $G(R)$ is Hamiltonian, then $L(G)$ is also Hamiltonian". Therefore $L(G(R))$ is Hamiltonian.

Theorem 2.12. Let $R$ be a finite commutative ring having $|U(R)| \leq 2$. Then $L(G(R))$ is planar.

Proof. Let $R$ be a finite commutative ring with unity such that $|U(R)| \leq 2$. Then in view of Proposition 2.8, $R$ is isomorphic to $\mathbf{Z}_{2}^{t} \times S(t \geq 0)$, where $S \cong \mathbf{Z}_{2}$ or $\mathbf{Z}_{3}$ or $\mathbf{Z}_{4}$ or $\mathbf{Z}_{6}$ or $\frac{\mathbf{Z}_{2}[x]}{\left\langle x^{2}\right\rangle}$. To show that $L(G(R))$ is planar, by following the steps analogues to those used in proof of Theorem 2.9, we found that if $R \cong \mathbf{Z}_{2}^{t} \times S$, then for $t=0$ and $S$ is either $\mathbf{Z}_{2}$ or $\mathbf{Z}_{3}$ or $\mathbf{Z}_{4}$ or $\frac{\mathbf{Z}_{2}[x]}{\left\langle x^{2}\right\rangle}$, or $\mathbf{Z}_{6}$ then $L(G(R))$ is isomorphic to $K_{1}$, $K_{2}, C_{4}, C_{4}$,and $C_{6}$ respectively, which are all planar.

On the other hand if $t \geq 1$ and $S$ is either $\mathbf{Z}_{2}$ or $\mathbf{Z}_{3}$ or $\mathbf{Z}_{4}$ or $\frac{\mathbf{Z}_{2}[x]}{\left\langle x^{2}\right\rangle}$, or $\mathbf{Z}_{6}$, then for each of the above $S, L(G(R))$ is isomorphic to $\cup_{i=1}^{2^{t}} K_{1}, \cup_{i=1}^{2^{t-1}} C_{6}, \cup_{i=1}^{2^{t}} C_{4}$, $\cup_{i=1}^{2^{t}} C_{4}$, and $\cup_{i=1}^{2^{t}} C_{6}$ respectively and is not difficult to verify that each of the listed line graph is planar. Thus the result follows.

Definition 2.4. The chromatic number of graph $G$, denoted by $\chi(G)$ is the minimum number of colors that can be assigned to the vertices in such a way that every two adjacent vertices have different color.

In the next theorem a new class of weakly perfect graph have been exposed using the tool of chromatic number and clique.

Theorem 2.13. Let $R$ be a finite commutative ring with unity and $U(R)$ be the set of units of $R$. Then $L(G(R))$ is weakly perfect.

Proof. Let $R$ be a finite commutative ring with unity and $U(R)$ be the set of units of $R$. In order to prove the desired result it is enough to show that $\chi(L(G(R)))=$ $\omega(L(G(R)))$. To do this we claim the followings:

i) $\chi(L(G(R)))=|U(R)|$ and $i i) \omega(L(G(R)))=|U(R)|$.

Towards proving $i$ ) it is known that the maximum degree of a vertex in $G(R)$ is $|U(R)|$, this implies that $\chi(L(G(R))) \geq|U(R)|$. Since the edge coloring in $G(R)$ leads to the vertex coloring in $L(G(R))$ and [2, Theorem 5.2] depicts that $G(R)$ is of class one. Therefore, $\chi(L(G(R)))=|U(R)|$.

ii) Note that the maximum degree of a vertex in $G(R)$ is $|U(R)|$ and therefore corresponding to that degree(of a vertex in $G(R)$ ) there exist a maximal clique of order $|U(R)|$ in $L(G(R))$. Thus $\omega(L(G(R)))=|U(R)|$. This indicates that $\chi(L(G(R)))=\omega(L(G(R)))=|U(R)|$, and hence $L(G(R))$ turns out to be weakly perfect.

Remark 2.14. In view of [6, Theorem 2.2] one can notice that $G(R)$ is weakly perfect graph, and Theorem 2.13 depicts that $L(G(R))$ is also weakly perfect, which is a enlargement of a new class of weakly perfect graphs, via. derived graphs. 


\section{References}

[1] A. Kumar, Pranjali, M. Acharya, and P. Sharma, "Unit graphs having their domination number half their order," in Recent advancements in graph theory, N. P. Shrimali and N. H. Shah, Eds. Boca Raton, FL: CRC Press, 2020, pp. 205-218.

[2] N. Ashrafi, H. R. Maimani, M. R. Pournaki, and S. Yassemi, “Unit graphs associated with rings", Communications in algebra, vol. 38, no. 8, pp. 2851-2871, 2010, doi: 10.1080/ 00927870903095574

[3] R. P. Grimaldi, "Graphs from rings”, Congressus numerantium, vol. 71, pp. 95103, 1990.

[4] F. Harary, Graph theory. Reading, MA: Addison-Wesley, 1969.

[5] N. Jacobson, Lectures in abstract algebra. New Delhi: East-West Press, 1951.

[6] H. R. Maimani, M. R. Pournaki, and S. Yassemi, "Weakly perfect graphs arising from rings", Glasgow mathematical journal, vol. 52, no. 3, pp. 417-425, 2010, doi: $10.1017 /$ S0017089510000108

[7] Pranjali, "Graphs associated with commutative rings", Ph.D. Thesis, University of Delhi, Department of Mathematics, 2016.

[8] Pranjali and M. Acharya, "Energy and Wiener index of unit graphs", Applied mathematics \& information sciences, vol. 9, no. 3, pp. 1339-1343, 2015. [On line]. Available: https:/ / bit.ly/ 3kBeS95 